\title{
Structure and EM form factors of purely relativistic systems
}

\author{
V.A. Karmanov* \\ Lebedev Physical Institute, Leninsky prospect 53, 11991 Moscow, Russia \\ E-mail: karmanovva@lebedev.ru
}

J. Carbonell

Université Paris-Saclay, CNRS/IN2P3, IJCLab, 91405 Orsay, France

E-mail: carbonelleipno.in2p3.fr

\section{H. Sazdjian}

Université Paris-Saclay, CNRS/IN2P3, IJCLab, 91405 Orsay, France

E-mail: sazdjian@ipno.in2p3.fr

\begin{abstract}
The Bethe-Salpeter equation for two massive scalar particles interacting by scalar massless exchange has solutions of two types, which differ from each other by their behavior in the nonrelativistic limit: the normal solutions which turn into the Coulomb ones and the "abnormal" solutions. The latter ones have no non-relativistic counterparts and disappear in the non-relativistic limit. We studied the composition of all these states. It turns out that the normal states, even for large binding energy, are dominated by two massive particles. Whereas, the contribution of the two-body sector into the abnormal states, even for small binding energy, is of the order of $1 \%$ only; they are dominated by an indefinite number of the massless particles. The elastic electromagnetic form factors for both normal and abnormal states, as well as the transition ones between them, are calculated.
\end{abstract}

Light Cone 2019 - QCD on the light cone: from hadrons to heavy ions - LC2019

16-20 September 2019

Ecole Polytechnique, Palaiseau, France

*Speaker. 


\section{Introduction}

In the Wick-Cutkosky model [1,2] (two scalar constituent particles interacting by massless scalar exchange) and for enough large coupling constant $\left(\alpha>\frac{\pi}{4}\right)$, there exist two different types of solutions of the Bethe-Salpeter (BS) equation [3]. In the non-relativistic limit (understood as the speed of light $c$, taken as a parameter, tending to infinity: $c \rightarrow \infty$ ), some solutions $\Phi(k, p)$ turn into the well-known solutions in the Coulomb potential; other solutions, on the contrary, disappear. The latter ones have purely relativistic origin and are called "abnormal". They can be odd or even relative to $k_{0} \rightarrow-k_{0}$. However, as shown in [4], the odd solutions do not contribute in the S-matrix and therefore, they are hardly physical ("observable"). The search for the abnormal symmetric states generated by massive exchange is in progress.

The following questions immediately arise: Are the abnormal states a consequence of a mathematical flaw of the BS equation? If not, how are they constructed, i.e. which constituents do they contain ? Why do they not have non-relativistic counterparts?

In this contribution, still in the Wick-Cutkosky model, i.e., for the massless exchange, we will study the physical nature of the abnormal states and answer these questions.

\section{Two-body contribution and EM form factors}

The state vector $|p\rangle$ is schematically represented via the Fock decomposition as follows:

$$
|p\rangle=\psi_{2}|2\rangle+\psi_{3}|3\rangle+\psi_{4}|4\rangle+\ldots
$$

where in the Wick-Cutkosky model the decomposition starts with the two-body state $|2\rangle$ containing two constituent particles only (each with mass $m$ ). Each higher state still contains two constituent particles, but with extra exchanged particles. The state vector $|p\rangle$ is normalized as

$$
\langle p \mid p\rangle=N_{2}+N_{3}+\ldots=1,
$$

where $N_{2}, N_{3}$ etc. are contributions of the two-, three-, etc. Fock sectors. Using the BS solution, we will be able to find $\psi_{2}$ and, correspondingly, $N_{2}$. To this aim, we will use the relation between the BS amplitude and $\psi_{2}$. It has the form (see, e.g., [5], Sec. 3.3):

$$
\psi_{2}\left(\vec{k}_{\perp}, x\right)=\frac{\left(\frac{1}{2} \omega \cdot p+\omega \cdot k\right)\left(\frac{1}{2} \omega \cdot p-\omega \cdot k\right)}{\pi(\omega \cdot p)} \int_{-\infty}^{\infty} \Phi(k+\beta \omega, p) d \beta
$$

We use here the explicitly covariant version of light-front dynamics [5], where the state vector is defined on the hyperplane $\omega \cdot x=0$, taking for $\omega=\left(\omega_{0}, \vec{\omega}\right)$ the four-vector with $\omega^{2}=0$, with an arbitrary orientation of $\vec{\omega}$. The standard version corresponds to the particular case $\omega=(1,0,0,-1)$. The BS amplitude $\Phi$ is normalized with the condition that the electromagnetic (em) form factor, expressed through it at $q=0$, is equal to 1 . This normalization is just equivalent to the condition (2.2).

This method was used in [6] to find the dependence of the two-body contribution $N_{2}$ to the normal ground state on the coupling constant $\alpha$. It was found that for small $\alpha$ (small binding energy $B \ll m$, total mass $M \approx 2 m) N_{2}$ is very close to 1 . For large $\alpha(\alpha \rightarrow 2 \pi$, the critical value, 
providing the solution with $M^{2} \rightarrow 0$ and the binding energy $\left.B \rightarrow 2 m\right) N_{2}$ tends to $9 / 14 \approx 64 \%$. Our consideration here is completely analogous to what was done in [6]. The only difference is that instead of the normal BS solution $\Phi(k, p)$, we take the abnormal one.

The BS solution is represented via the weight function $g(z)$ in the form of Nakanishi integral representation [7]

$$
\Phi(k ; p)=\frac{i}{\sqrt{N_{t o t}}} \int_{-1}^{1} \frac{g(z) d z}{\left(k^{2}+p \cdot k z+\frac{1}{4} M^{2}-m^{2}+i \varepsilon\right)^{3}} .
$$

The normalization factor $N_{t o t}$ will be found below.

The function $g(z)$ satisfies a differential equation $[1,2]$ and is labelled by two quantum numbers $n, k$. The states with $k=0$ are the normal ones. In the non-relativistic limit the binding energies reproduce the Coulomb spectrum $B_{n}=2 m-M=\frac{\alpha^{2} m}{4 n^{2}}$. The states with $k \neq 0$ are the abnormal ones. The value of $k$ corresponds to the number of nodes of the function $g(z)$. For even $k$ the function $g(z)$ is symmetric relative to $z \rightarrow-z$, for odd $k$ it is antisymmetric. We will consider the solutions with $n=1$ and $k=0,2,4$. Then the equation for $g(z)$ obtains the form:

$$
g^{\prime \prime}(z)+\frac{\alpha}{\pi} \frac{1}{\left(1-z^{2}\right)\left(1-\eta^{2}+\eta^{2} z^{2}\right)} g(z)=0,
$$

where $\eta=\frac{M}{2 m}=1-\frac{B}{2 m}$ and the boundary conditions are $g( \pm 1)=0$. We solved this equation numerically, generating the spectrum labelled by $k$.

Substituting $\Phi(k ; p)$ from (2.4) into (2.3), we find $\psi_{2}\left(\vec{k}_{\perp}, x\right)$ in terms of $g(z)$ :

$$
\psi\left(\vec{k}_{\perp}, x\right)=\frac{1}{\sqrt{N_{\text {tot }}}} \frac{x(1-x) g(1-2 x)}{\left[\vec{k}_{\perp}^{2}+m^{2}-x(1-x) M^{2}\right]^{2}} .
$$

In this way, we find the two-body contribution into the full norm (equaled to 1):

$$
N_{2}=\langle 2 \mid 2\rangle=\frac{1}{(2 \pi)^{3}} \int \psi_{n}^{2}\left(\vec{k}_{\perp}, x\right) \frac{d^{2} k_{\perp} d x}{2 x(1-x)}=\frac{1}{48 \pi^{2} N_{t o t}} \int_{0}^{1} \frac{x(1-x) g^{2}(1-2 x) d x}{\left[m^{2}-x(1-x) M^{2}\right]^{3}} .
$$

The elastic electromagnetic form factors can be also expressed via $g(z)[6]$ :

$$
\begin{aligned}
F(Q) & =\frac{1}{8 \pi^{2} N_{t o t}} \int_{0}^{1} g(1-2 x) d x \int_{0}^{1} g\left(1-2 x^{\prime}\right) d x^{\prime} \\
& \times \int_{0}^{1} d u u^{2}(1-u)^{2} \frac{\left[x x^{\prime} u(1-u) Q^{2}+(6 \xi-5) m^{2}+2 M^{2} \xi(1-\xi)\right]}{\left[x x^{\prime} u(1-u) Q^{2}+m^{2}-\xi(1-\xi) M^{2}\right]^{4}},
\end{aligned}
$$

where $\xi=(1-x) u+\left(1-x^{\prime}\right)(1-u)$ and $Q^{2}=-q^{2}=-\left(p-p^{\prime}\right)^{2}>0$. The constant $N_{t o t}$ is found from the condition $F(0)=1$. The transition form factor (between two different states) was calculated according to ref. [8].

\section{Numerical results}

We solved numerically Eq. (2.5) for $g(z)$, taking $m=1$, for a few values of $\alpha$ in the interval $\alpha=0.02 \div 5$. As an example, in Fig. 1 the solutions $g(z)$ for $\alpha=5, n=1$ and $k=0,2,4$ are shown. 

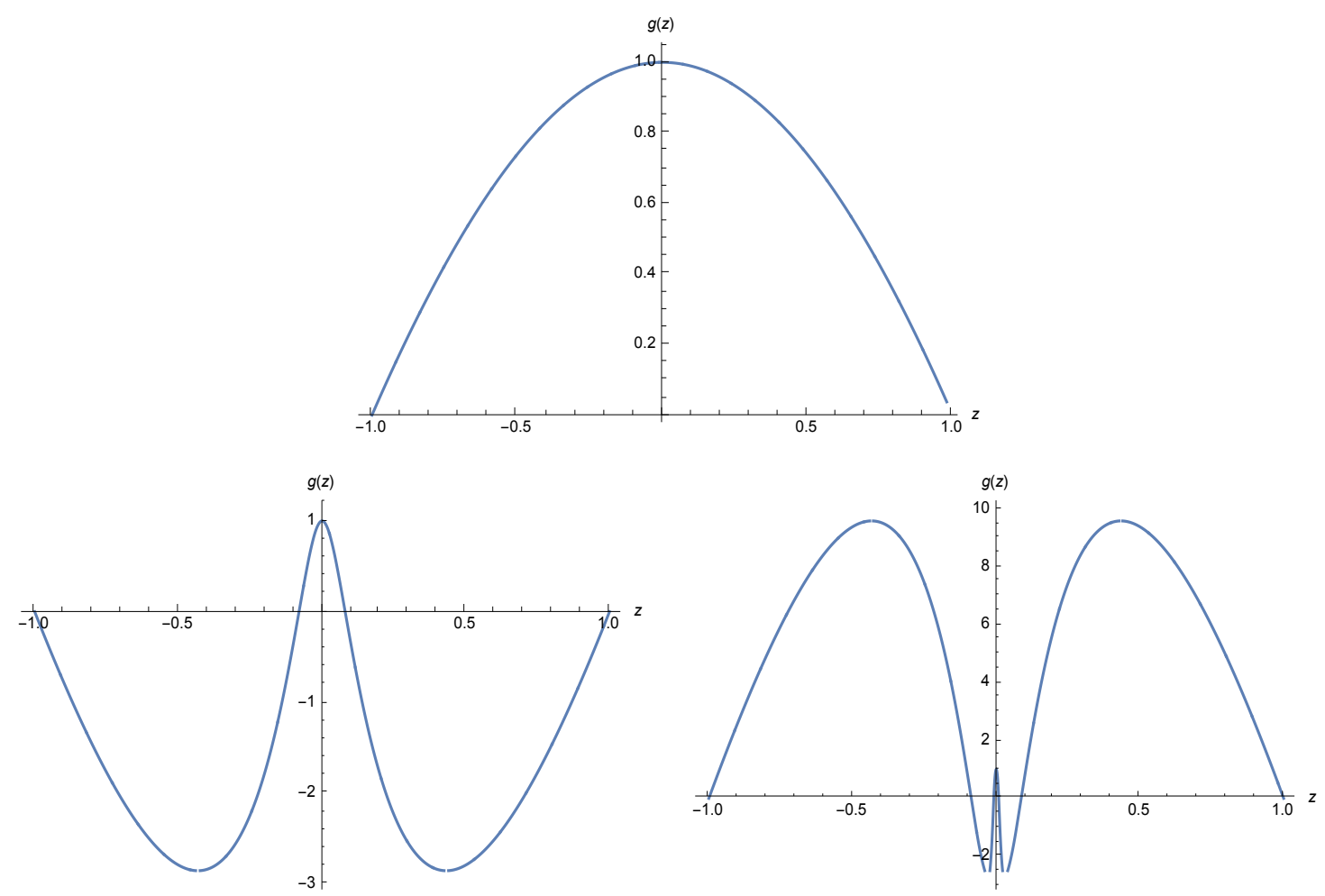

Figure 1: The solutions $g(z)$ (normalized by $g(0)=1$ ) of Eq. (2.5) for $\alpha=5, n=1$ and $k=0$ (at the top, normal, zero nodes), $k=2$ (at the bottom left, abnormal, two nodes) and $k=4$ (at the bottom right, abnormal, four nodes).

\begin{tabular}{clcll}
\hline No. & $\alpha$ & $k=N_{\text {nodes }}$ & $B$ & $N_{2}$ \\
\hline 1 & 0.02 & 0 & 0.000091 & 0.96 \\
2 & 0.2 & 0 & 0.006271 & 0.85 \\
3 & 2 & 0 & 0.236 & 0.70 \\
4 & 2 & 2 & $0.975 \cdot 10^{-5}$ & $7.7 \cdot 10^{-3}$ \\
5 & 5 & 0 & 0.999 & 0.65 \\
6 & 5 & 2 & $3.512 \cdot 10^{-3}$ & $9.35 \cdot 10^{-2}$ \\
7 & 5 & 4 & $1.55 \cdot 10^{-5}$ & $6.14 \cdot 10^{-3}$ \\
\hline
\end{tabular}

Table 1: Two-body contributions $N_{2}$ into the full normalization integral (equal to 1) for some coupling constants and corresponding binding energies.

Using these solutions, we calculated by Eq. (2.7) the two-body contributions $N_{2}$ to the total norm for a few low-lying states with $n=1$, both normal $(k=0)$ and symmetric abnormal $(k=2,4)$. The results are given in Table 1. For the abnormal states they are shown in red.

For the normal states with $\alpha \ll 1$, the binding energy $B$ is close to the Coulombean value $\frac{1}{4} \alpha^{2}$, through at $\alpha=0.2$ the relativistic correction is large $(\approx 40 \%)$. Besides, the values of $N_{2}$ vary in the "normal" interval between (for small $\left.\alpha=10^{-4}\right) N_{2}=0.99$ (the limiting case $N_{2}(\alpha \rightarrow 0) \rightarrow 1$ ) 
and (for large $\alpha=5$ ) $N_{2}=0.65$ (the limiting case $N_{2}(\alpha \rightarrow 2 \pi) \rightarrow 0.64$ ).

Whereas, for the abnormal states $\left(k=N_{\text {nodes }}=2\right.$ and 4$)$, even with very small binding energies, the two-body contribution is of the order of a few per cent only. This means that the abnormal states are mainly many-body ones (two constituents with mass $m+$ a few or many exchanged massless particles). This explains their disappearance in the non-relativistic limit.
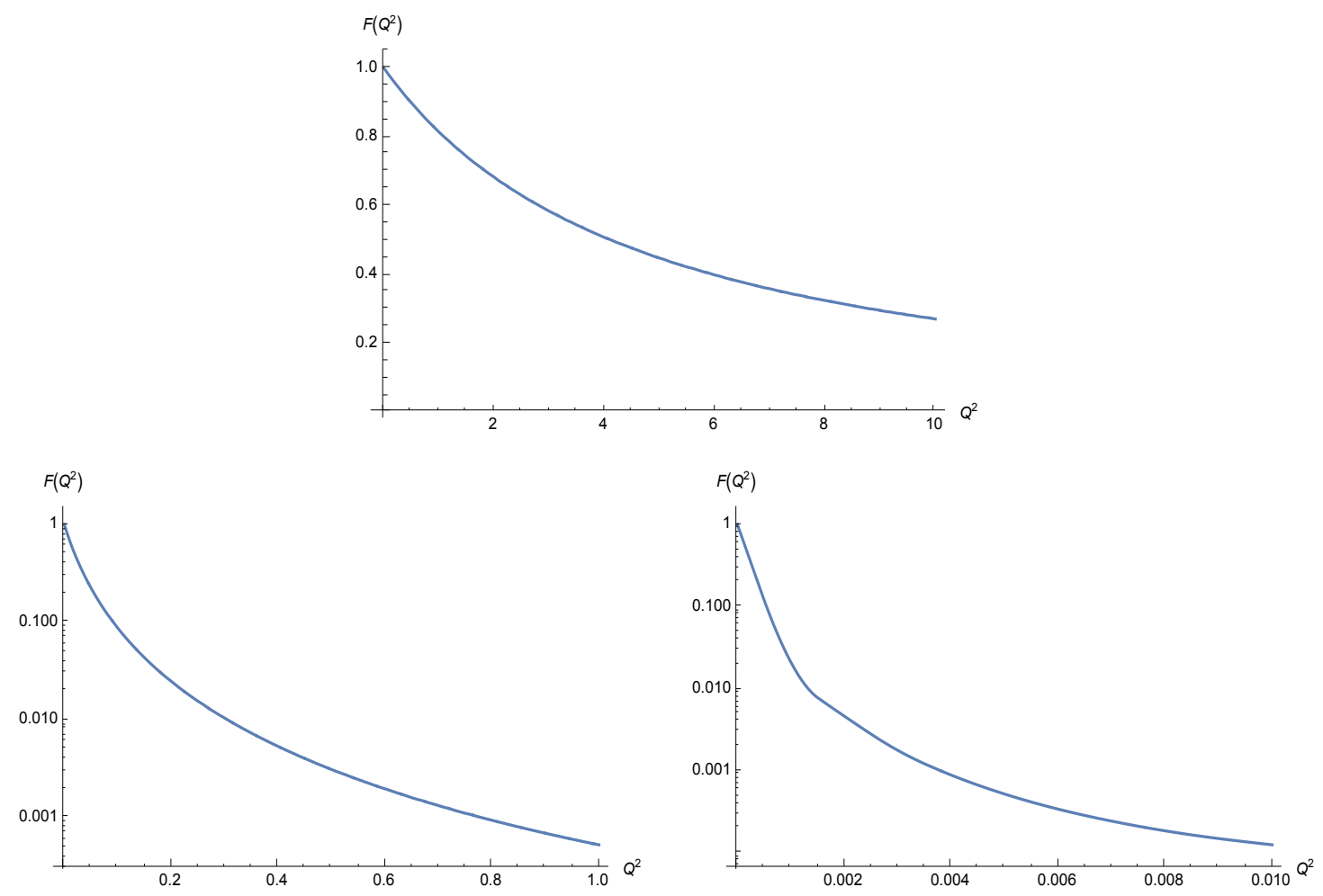

Figure 2: Top, bottom left and bottom right are the elastic form factors of the states No. 5 (normal), 6 and 7 (both abnormal) from the Table 1 (calculated correspondingly with the top, bottom left and bottom right $g(z)$ 's shown in Fig. 1).

Figure 2 shows that the elastic em form factors of abnormal state with two nodes (bottom left) vs. $Q^{2}$ decrease, at least, $\sim 1000$ times faster than those of the normal states (top), as should be for a many-body system. The same for the abnormal state with four nodes (bottom right) decreases even more faster in shorter interval of $Q^{2}$.

Figure 3 shows that the transition form factors between the normal and abnormal states (top left and right) are one-two orders of magnitude smaller than those between the abnormal ones (bottom). In this sense, the normal and abnormal states represent different "worlds", weakly "communicating" with each other.

These observations shed light on the nature and properties of the abnormal states.

\section{Conclusion}

The normal and abnormal states of the Bethe-Salpeter equation drastically differ by their Fockspace content. In the normal states the two-body (constituent) contribution dominates, whereas the 

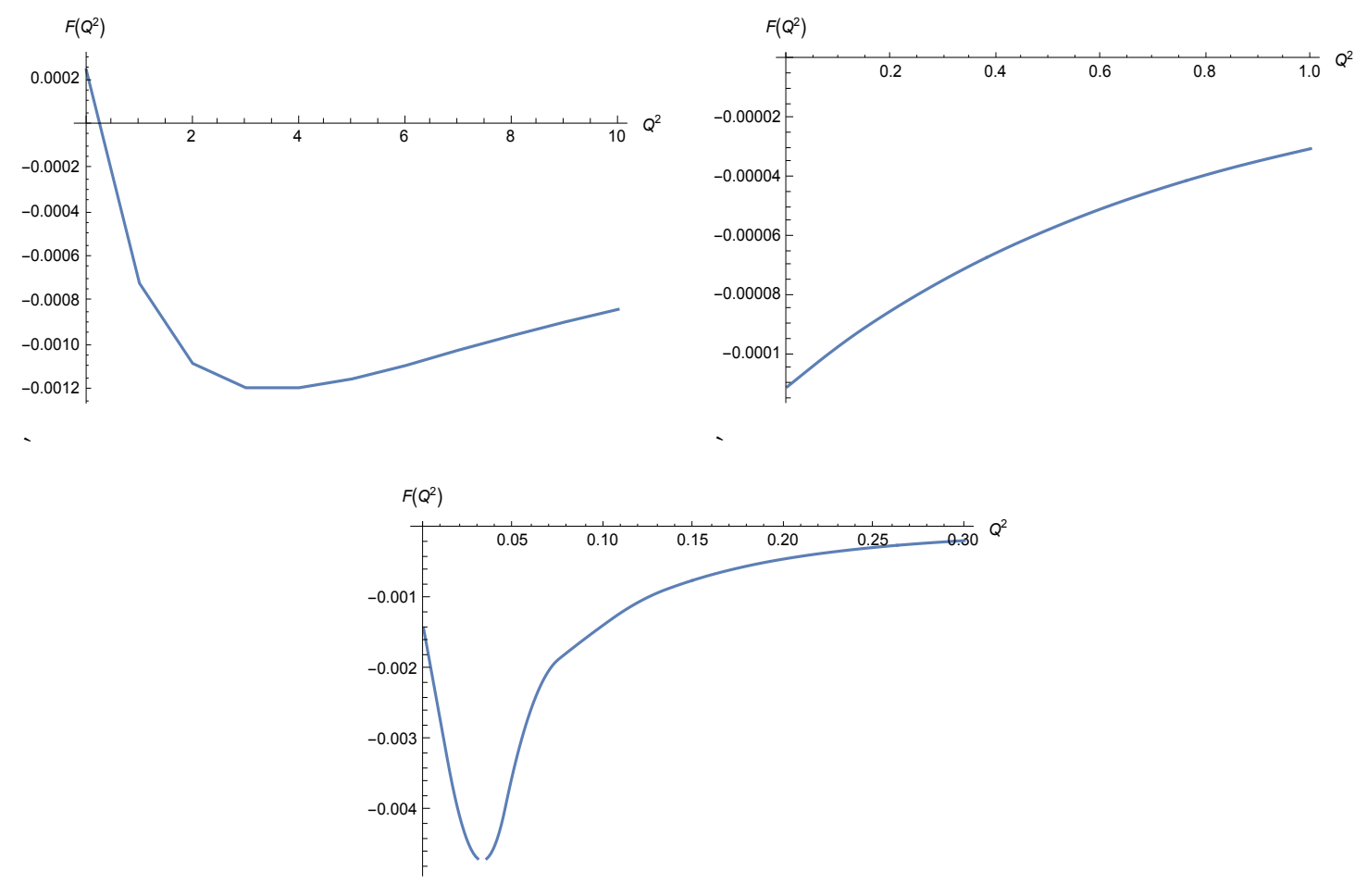

Figure 3: Top left is the transition form factor between the (normal) state No. 5 from the Table 1 and the abnormal one with two nodes No. 6; top right is the transition form factor between the state No. 5 and the abnormal one with four nodes No. 7; bottom is the transition form factor between the two abnormal states No. 6 and 7.

abnormal ones are dominated by the many-body contributions of the exchanged particles.

\section{References}

[1] G.C. Wick, Properties of Bethe-Salpeter Wave Functions, Phys. Rev. 96 (1954) 1124.

[2] R.E. Cutkosky, Solutions of a Bethe-Salpeter Equation, Phys. Rev. 96 (1954) 1135.

[3] E.E. Salpeter, H. Bethe, A Relativistic Equation for Bound-State Problems, Phys. Rev. 84 (1951) 1232.

[4] M. Ciafaloni and P. Menotti, Operator Analysis of the Bethe-Salpeter Equation, Phys. Rev. 140 (1965) B929.

[5] J. Carbonell, B. Desplanques, V.A. Karmanov, J.-F. Mathiot, Explicitly Covariant Light-Front Dynamics and Relativistic Few-Body Systems, Phys. Rep., 300 (1998) 215 [nucl-th/ 9804029 ].

[6] Dae Sung Hwang and V.A. Karmanov, Many-body Fock sectors in Wick-Cutkosky model, Nucl. Phys. B 696 (2004) 413 [hep-th/ 0405035$].$

[7] N. Nakanishi, Partial-Wave Bethe-Salpeter Equation, Phys. Rev. 130 (1963) 1230; Graph Theory and Feynman Integrals, Gordon and Breach, New-York, 1971.

[8] J. Carbonell, V.A. Karmanov, Transition electromagnetic form factor and current conservation in the Bethe-Salpeter approach, Phys. Rev. D 91 (2015) 076010 [1504.02450]. 